\title{
SURVEY ARTICLE: SIMPLICIAL COMPLEXES SATISFYING SERRE'S CONDITION: A SURVEY WITH SOME NEW RESULTS
}

\author{
M.R. POURNAKI, S.A. SEYED FAKHARI, N. TERAI AND S. YASSEMI \\ Dedicated with gratitude to our friend Richard P. Stanley \\ on the occasion of his 70 th birthday
}

\begin{abstract}
The problem of finding a characterization of Cohen-Macaulay simplicial complexes has been studied intensively by many authors. There are several attempts at this problem available for some special classes of simplicial complexes satisfying some technical conditions. This paper is a survey, with some new results, of some of these developments. The new results about simplicial complexes with Serre's condition are an analogue of the known results for Cohen-Macaulay simplicial complexes.
\end{abstract}

1. Preliminaries. Algebraic combinatorics is an area of mathematics that employs methods of abstract algebra in various combinatorial contexts and vice versa. One of the fastest developing subfields within algebraic combinatorics is combinatorial commutative algebra. It has evolved into one of the most active areas of mathematics during the past several decades. We refer the reader to the books by Stanley [36], Bruns and Herzog [3], Miller and Sturmfels [25], as well as Herzog and Hibi [16] as general references in the subject.

2010 AMS Mathematics subject classification. Primary 13C15, 05E99, Secondary $13 \mathrm{C} 13$.

Keywords and phrases. Simplicial complex, Cohen-Macaulay simplicial complex, $\left(S_{r}\right)$ simplicial complex, sequentially Cohen-Macaulay simplicial complex, sequentially $\left(S_{r}\right)$ simplicial complex.

The research of the first, second, and fourth authors was in part supported by a grant from IPM (Nos. 92130115, 92130422 and 92130214). This work has been also partially supported by the Center for International Scientific Studies and Collaboration (CISSC), Iran, and Égide-Programme Gundishapur 2012, Hubert Curien Partnership, France (No. 27462PL).

Received by the editors on October 27, 2012, and in revised form on October 29, 2013. 
A simplicial complex $\Delta$ on the set of vertices $[n]:=\{1, \ldots, n\}$ is a collection of subsets of $[n]$ which is closed under taking subsets; that is, if $F \in \Delta$ and $F^{\prime} \subseteq F$, then also $F^{\prime} \in \Delta$. Every element $F \in \Delta$ is called a face of $\Delta$; the size of a face $F$ is defined to be $|F|$ and its dimension is defined to be $|F|-1$. (As usual, for a given finite set $X$, the number of elements of $X$ is denoted by $|X|$.) The dimension of $\Delta$, which is denoted by $\operatorname{dim} \Delta$, is defined to be $d-1$, where $d=\max \{|F| \mid F \in \Delta\}$. A facet of $\Delta$ is a maximal face of $\Delta$ with respect to inclusion. Let $\mathcal{F}(\Delta)$ denote the set of facets of $\Delta$. It is clear that $\mathcal{F}(\Delta)$ determines $\Delta$. When $\mathcal{F}(\Delta)=\left\{F_{1}, \ldots, F_{m}\right\}$, we write $\Delta=\left\langle F_{1}, \ldots, F_{m}\right\rangle$. We say that $\Delta$ is pure if all facets of $\Delta$ have the same cardinality. The link of $\Delta$ with respect to a face $F \in \Delta$, denoted by $\operatorname{lk}_{\Delta}(F)$, is the simplicial complex $\mathrm{lk}_{\Delta}(F)=\{G \subseteq[n] \backslash F \mid G \cup F \in \Delta\}$, and the deletion of $F$, denoted by $\operatorname{del}_{\Delta}(F)$, is the simplicial complex $\operatorname{del}_{\Delta}(F)=\{G \subseteq[n] \backslash F \mid G \in \Delta\}$. When $F=\{x\}$ is a single vertex, we abuse notation and write $\operatorname{lk}_{\Delta}(x)$ and $\operatorname{del}_{\Delta}(x)$. For a given simplicial complex $\Delta$ on $[n]$, we define $\Delta^{\vee}$ by $\Delta^{\vee}=\{[n] \backslash F \mid F \notin \Delta\}$. The simplicial complex $\Delta^{\vee}$ is called the Alexander dual of $\Delta$.

One of the connections between combinatorics and commutative algebra is via rings constructed from the combinatorial objects. Let $R=\mathbb{K}\left[x_{1}, \ldots, x_{n}\right]$ be the polynomial ring in $n$ variables over a field $\mathbb{K}$, and let $\Delta$ be a simplicial complex on $[n]$. For every subset $F \subseteq[n]$, we set $x_{F}=\prod_{i \in F} x_{i}$. The Stanley-Reisner ideal of $\Delta$ over $\mathbb{K}$ is the ideal $I_{\Delta}$ of $R$ which is generated by those squarefree monomials $x_{F}$ with $F \notin \Delta$. In other words, $I_{\Delta}=\left\langle x_{F} \mid F \in \mathcal{N}(\Delta)\right\rangle$, where $\mathcal{N}(\Delta)$ denotes the set of minimal nonfaces of $\Delta$ with respect to inclusion. The Stanley-Reisner ring of $\Delta$ over $\mathbb{K}$, denoted by $\mathbb{K}[\Delta]$, is defined to be $\mathbb{K}[\Delta]=R / I_{\Delta}$. Let $I \subseteq R$ be an arbitrary squarefree monomial ideal. Then there is a unique simplicial complex $\Delta$ such that $I=I_{\Delta}$. For simplicity, we often write $I^{\vee}$ to denote the ideal $I_{\Delta \vee}$, and we call it the Alexander dual of $I$.

We finish these preliminaries with the following well-known characterization problem. We first recall two notions from commutative algebra: Cohen-Macaulayness and satisfying Serre's condition. In mathematics, Cohen-Macaulay rings are particular types of commutative rings, possessing some of the algebraic-geometric properties of nonsingular varieties, such as local equidimensionality. These rings are named after Francis Sowerby Macaulay (1862-1937), who proved the 
unmixedness theorem for polynomial rings in 1916, and Irvin Sol Cohen (1917-1955), who proved the unmixedness theorem for formal power series rings in 1946. All Cohen-Macaulay rings have the unmixedness property. Let $R=\mathbb{K}\left[x_{1}, \ldots, x_{n}\right]$ and $M$ be a nonzero finitely generated $R$-module. We say that $M$ is Cohen-Macaulay if, for every $\mathfrak{p} \in \operatorname{Spec}(\mathrm{R})$, the equality depth $M_{\mathfrak{p}}=\operatorname{dim} M_{\mathfrak{p}}$ holds true. Also, $M$ is said to satisfy Serre's condition $\left(S_{r}\right)$, or simply $M$ is an $\left(S_{r}\right)$ module if, for every $\mathfrak{p} \in \operatorname{Spec}(\mathrm{R})$, the inequality depth $M_{\mathfrak{p}} \geq \min \left\{r, \operatorname{dim} M_{\mathfrak{p}}\right\}$ holds true. It is easy to see that $M$ is Cohen-Macaulay if and only if it is an $\left(S_{r}\right)$ module for all $r \geq 1$. We say that a simplicial complex $\Delta$ is Cohen-Macaulay over a field $\mathbb{K}$, if the Stanley-Reisner ring $\mathbb{K}[\Delta]$ of $\Delta$ is Cohen-Macaulay. Also, $\Delta$ is said to satisfy Serre's condition $\left(S_{r}\right)$ over a field $\mathbb{K}$, or simply $\Delta$ is an $\left(S_{r}\right)$ simplicial complex over $\mathbb{K}$, if the Stanley-Reisner ring $\mathbb{K}[\Delta]$ of $\Delta$ satisfies Serre's condition $\left(S_{r}\right)$. Since every simplicial complex satisfies Serre's condition $\left(S_{1}\right)$, throughout this paper we assume that $r \geq 2$. It is well known that if $\Delta$ is an $\left(S_{r}\right)$ simplicial complex over a field $\mathbb{K}$, then $\Delta$ is pure (see [28, Lemma $2.6])$.

Problem 1.1. Find a characterization of Cohen-Macaulay simplicial complexes.

There are several attempts at this problem available for some special classes of simplicial complexes satisfying some technical conditions. This paper is a survey, with some new results, of some of these developments. The new results about simplicial complexes with Serre's condition are analogues of the known results for Cohen-Macaulay simplicial complexes.

2. Reisner's criterion. Let $\mathbb{K}$ be a field and $\Delta$ a simplicial complex of dimension $d-1$ with the vertex set $V=\left\{v_{1}, \ldots, v_{n}\right\}$. We write $C_{i}=C_{i}(\Delta ; \mathbb{K}),-1 \leq i \leq d-1$, for the vector space over $\mathbb{K}$ with basis which consists of all $i$-dimensional faces of $\Delta$. For $i$ with $0 \leq i \leq d-1$, we now define a linear map $\partial_{i}: C_{i} \rightarrow C_{i-1}$ (by defining it on the basis elements) as follows: for an $i$-dimensional face $F$ of $\Delta$ whose vertices 
are $v_{l_{0}}, v_{l_{1}}, \ldots, v_{l_{i}}$, where $1 \leq l_{0}<l_{1}<\cdots<l_{i} \leq n$, consider

$$
\partial_{i}(F)=\sum_{j=0}^{i}(-1)^{j}\left(F \backslash\left\{v_{l_{j}}\right\}\right) .
$$

By this definition we obtain the following sequence of linear maps:

$$
0 \longrightarrow C_{d-1} \stackrel{\partial_{d-1}}{\longrightarrow} C_{d-2} \longrightarrow \cdots \longrightarrow C_{1} \stackrel{\partial_{1}}{\longrightarrow} C_{0} \stackrel{\partial_{0}}{\longrightarrow} C_{-1} \longrightarrow 0 .
$$

One can easily check that, for every $1 \leq i \leq d-1, \partial_{i-1} \partial_{i}=0$. Therefore, the above sequence is actually a complex. The quotient vector space $\widetilde{H}_{i}(\Delta ; \mathbb{K})=\operatorname{Ker} \partial_{i} / \operatorname{Im} \partial_{\mathrm{i}+1}$ is called the $i$ th reduced homology group of $\Delta$ with coefficients in $\mathbb{K}$. We refer the reader to [27] for details concerning simplicial homology.

The following characterization of when a simplicial complex is Cohen-Macaulay is due to Reisner [30].

Theorem 2.1 (Reisner's criterion). Let $\mathbb{K}$ be a field and $\Delta$ a simplicial complex of dimension $d-1$. Then $\Delta$ is Cohen-Macaulay over $\mathbb{K}$ if and only if, for every $F \in \Delta$ and for every $i<\operatorname{dim}\left(\operatorname{lk}_{\Delta}(F)\right)$, $\widetilde{H}_{i}\left(\mathrm{lk}_{\Delta}(F) ; \mathbb{K}\right)=0$ holds true.

Reisner's criterion says that $\Delta$ is Cohen-Macaulay over the field $\mathbb{K}$ if and only if the homology of each face's link vanishes below its top dimension. A result due to Munkres then shows that the CohenMacaulayness of $\Delta$ over $\mathbb{K}$ is a topological property: it depends only on the homeomorphism class of the simplicial complex $\Delta$.

There are some results analogous to Reisner's criterion for some other algebraic properties like Buchsbaumness and Gorensteinness, which are omitted in this survey. We refer the interested reader to $[3,36]$ and we continue the survey with Serre's condition.

Using a result of Schenzel [33, Lemma 3.2.1] and also Hochster's formula on local cohomology modules, Terai has formulated the analogue of Reisner's criterion for $\left(S_{r}\right)$ simplicial complexes.

Theorem 2.2 ([38], Theorem 1.4 ff.). Let $\mathbb{K}$ be a field and $\Delta$ a simplicial complex of dimension $d-1$. Then $\Delta$ is $\left(S_{r}\right)$ over $\mathbb{K}$ if and 
only if, for every $F \in \Delta$ (including $F=\emptyset)$ with $|F| \leq d-i-2$ and for every $-1 \leq i \leq r-2, \widetilde{H}_{i}\left(\mathrm{lk}_{\Delta}(F) ; \mathbb{K}\right)=0$ holds true.

The above characterization leads to the following corollary.

Corollary 2.3 ([11], Lemma 2.2). Let $\mathbb{K}$ be a field and $\Delta$ a simplicial complex of dimension $d-1$. If $\Delta$ is $\left(S_{r}\right)$ over $\mathbb{K}$, then for every $F \in \Delta$ the simplicial complex $\mathrm{lk}_{\Delta}(F)$ is also $\left(S_{r}\right)$ over $\mathbb{K}$.

Let us recall the notion of connectedness. A simplicial complex $\Delta$ is called connected if, for every pair of facets $(F, G)$ of $\Delta$, there exists a sequence of facets $F=F_{0}, \ldots, F_{m}=G$ such that, for every $0 \leq i \leq m-1$, we have $F_{i} \cap F_{i+1} \neq \emptyset$.

The following corollary is an immediate consequence of Theorem 2.2.

Corollary 2.4. Let $\mathbb{K}$ be a field and $\Delta$ a simplicial complex. Then $\Delta$ is $\left(S_{2}\right)$ over $\mathbb{K}$ if and only if, for every face $F \in \Delta$ with $\operatorname{dim}\left(\mathrm{lk}_{\Delta}(F)\right) \geq 1$, the simplicial complex $\operatorname{lk}_{\Delta}(F)$ is connected. In particular, the $\left(S_{2}\right)$ property of a simplicial complex is independent from the base field.

There is a classical example of Reisner [30] of a triangulation of the real projective plane which is Cohen-Macaulay if Char $(\mathbb{K}) \neq 2$, but it is not Cohen-Macaulay if Char $(\mathbb{K})=2$. This shows that Cohen-Macaulayness of a simplicial complex is not independent from the base field. Comparing this fact with Corollary 2.4 leads us to ask the following question.

Question 2.5. Let $\Delta$ be a $(d-1)$-dimensional simplicial complex. For which integers $3 \leq r \leq d-1$ is the $\left(S_{r}\right)$ property of $\Delta$ independent from the base field?

We close this section by the following proposition. First, we recall two definitions. Let $\Delta$ be a simplicial complex. The simplicial complex $\Delta^{(i)}:=\{F \in \Delta \mid \operatorname{dim} F \leq i\}$ is called the $i$-skeleton of $\Delta$. Also, the simplicial complex $\Delta^{[i]}:=\langle F \in \Delta \mid \operatorname{dim} F=i\rangle$ is called the $i$-pure skeleton of $\Delta$. 
Proposition 2.6 ([11], Proposition 2.3). Let $\mathbb{K}$ be a field and $\Delta$ a simplicial complex. If $\Delta$ is $\left(S_{r}\right)$ over $\mathbb{K}$, then $\Delta^{(i)}$ is also $\left(S_{r}\right)$ over $\mathbb{K}$ $(2 \leq r \leq i+1)$.

3. Shellable simplicial complexes. According to the standard definition, a pure simplicial complex is shellable if its facets can be ordered so that they satisfy a simple combinatorial condition. A shellable simplicial complex is a special kind of a Cohen-Macaulay complex. Shellability is a notion familiar to commutative algebraists but one which has not received as much attention from combinatorialists. This notion is a simple but powerful tool for proving the Cohen-Macaulay property, and almost all Cohen-Macaulay complexes arising in nature turn out to be shellable. Björner and Wachs [2] dropped the requirement of purity from the definition and explored a more general concept of shellability. Here, we recall the notion of shellability. A simplicial complex $\Delta$ is called shellable if its facets can be arranged in linear order $F_{1}, F_{2}, \ldots, F_{t}$ in such a way that the subcomplex $\left\langle F_{1}, \ldots, F_{k-1}\right\rangle \cap\left\langle F_{k}\right\rangle$ is pure and has dimension $\operatorname{dim} F_{k}-1$ for every $k$ with $2 \leq k \leq t$. Such an ordering of facets is called a shelling order.

The following result, based on shellability and due to Hochster, is a well known combinatorial criterion for verifying that a pure simplicial complex is Cohen-Macaulay (see [16, Theorem 8.2.6] as a more accessible reference).

Theorem 3.1. Every pure shellable simplicial complex is CohenMacaulay over every field.

We are now in a position to state and prove the first new result of this paper.

Theorem 3.2. Let $\mathbb{K}$ be a field and $\Delta$ a simplicial complex. Then the following conditions are equivalent:

(a) There exists an ordering of facets of $\Delta$, say $F_{0}, \ldots, F_{m}$, such that, for every $0 \leq i \leq m$, the simplicial complex $\left\langle F_{0}, \ldots, F_{i}\right\rangle$ is Cohen-Macaulay over $\mathbb{K}$. 
(b) There exists an ordering of facets of $\Delta$, say $F_{0}, \ldots, F_{m}$, such that, for every $0 \leq i \leq m$, the simplicial complex $\left\langle F_{0}, \ldots, F_{i}\right\rangle$ is $\left(S_{2}\right)$.

(c) $\Delta$ is pure, and there exists an ordering of facets of $\Delta$, say $F_{0}, \ldots, F_{m}$, such that, for every $0 \leq i \leq m$, every link of $\left\langle F_{0}, \ldots, F_{i}\right\rangle$ with dimension at least one is connected.

(d) $\Delta$ is pure shellable.

Proof. The implications $(\mathrm{d}) \Rightarrow(\mathrm{a})$ and $(\mathrm{a}) \Rightarrow(\mathrm{b})$ are trivial. The implication $(\mathrm{b}) \Rightarrow(\mathrm{c})$ follows from Corollary 2.4. We now prove that (c) implies (d).

Let $\Delta$ be a pure simplicial complex of dimension $d-1$. If $d=1$, then $\Delta$ is a zero-dimensional simplicial complex, and thus it is pure shellable. Hence, we assume that $d \geq 2$ and suppose that there exists an ordering of facets of $\Delta$, say $F_{0}, \ldots, F_{m}$, such that, for every $0 \leq i \leq m$, every link of $\left\langle F_{0}, \ldots, F_{i}\right\rangle$ with dimension at least one is connected. We prove that this ordering is, in fact, a shelling order, and this proves (d). For this, it is enough to prove that, for every $1 \leq i \leq m,\left\langle F_{0}, \ldots, F_{i-1}\right\rangle \cap\left\langle F_{i}\right\rangle$ is pure of dimension $d-2$. Now suppose, on the contrary, that there exists $1 \leq i \leq m$ such that $\left\langle F_{0}, \ldots, F_{i-1}\right\rangle \cap\left\langle F_{i}\right\rangle$ is not pure of dimension $d-2$. This implies that $\left\langle F_{0}, \ldots, F_{i-1}\right\rangle \cap\left\langle F_{i}\right\rangle$ has a facet, say $G$, with dimension at most $d-3$. Note that $G$ is a face of $\Delta_{i}:=\left\langle F_{0}, \ldots, F_{i}\right\rangle$.

We now claim that $\operatorname{lk}_{\Delta_{i}}(G)$ is not connected. In order to prove the claim, note that $F_{i} \backslash G$ is a facet of $\operatorname{lk}_{\Delta_{i}}(G)$. The vertices of $F_{i} \backslash G$ do not appear in the other facets of $\operatorname{lk}_{\Delta_{i}}(G)$, because if $a$ is a vertex of $F_{i} \backslash G$ which appears also in another facet, say $G^{\prime}$, of $\operatorname{lk}_{\Delta_{i}}(G)$, then since $G^{\prime}$ and $F_{i} \backslash G$ are different facets of $\operatorname{lk}_{\Delta_{i}}(G)$, therefore $G^{\prime}$ is not a subset of $F_{i} \backslash G$, and so $G^{\prime} \cup G$ is not a subset of $F_{i}$. In particular, $G^{\prime} \cup G \neq F_{i}$. Since $G^{\prime}$ is a facet of $\operatorname{lk}_{\Delta_{i}}(G), G^{\prime} \cup G$ is a facet of $\Delta_{i}$ and since $G^{\prime} \cup G \neq F_{i}$, therefore there exists $0 \leq j \leq i-1$ such that $G^{\prime} \cup G=F_{j}$. Now, $G \cup\{a\} \subseteq F_{i}$ and, since $\bar{a} \in \bar{G}^{\prime}$, therefore $G \cup\{a\} \subseteq G^{\prime} \cup G=F_{j}$, and so $G \cup\{a\}$ is a facet of $\left\langle F_{0}, \ldots, F_{i-1}\right\rangle \cap\left\langle F_{i}\right\rangle$ and it properly contains $G$. This is a contradiction, because $G$ is a facet of $\left\langle F_{0}, \ldots, F_{i-1}\right\rangle \cap\left\langle F_{i}\right\rangle$. Therefore, the vertices of $F_{i} \backslash G$ do not appear in the other facets of $\mathrm{lk}_{\Delta_{i}}(G)$, and so there does not exist any path in $\mathrm{lk}_{\Delta_{i}}(G)$ that connects $F_{i} \backslash G$ to other facets of $\mathrm{lk}_{\Delta_{i}}(G)$ and therefore 
$\mathrm{lk}_{\Delta_{i}}(G)$ is not connected. This completes the proof of the claim.

Note that, since $\operatorname{dim} G \leq d-3$, we conclude that $\operatorname{dim} \mathrm{lk}_{\Delta_{i}}(G) \geq 1$, and this contradicts (c).

As an analogue for Theorem 3.2, we state and prove the following result.

Theorem 3.3. Let $\mathbb{K}$ be a field and $\Delta a(d-1)$-dimensional simplicial complex. Suppose that $\Delta=\Delta_{0} \cup\langle F\rangle$, where $\Delta_{0}$ is a $(d-1)$-dimensional subcomplex of $\Delta$ which is $\left(S_{r}\right)$ over $\mathbb{K}$ and $F$ is a $(d-1)$-dimensional facet of $\Delta$ such that $F \notin \Delta_{0}$. Then $\Delta$ is $\left(S_{r}\right)$ over $\mathbb{K}$ if and only if it is $\left(S_{2}\right)$.

Proof. For $d \leq 2$ there is nothing to prove, since in this case the concept of $\left(S_{r}\right)$ and $\left(S_{2}\right)$ coincide. We therefore assume $d \geq 3$. Since every $\left(S_{r}\right)$ simplicial complex is $\left(S_{2}\right)$, it is enough to prove that, if $\Delta$ is an $\left(S_{2}\right)$ simplicial complex, then it is $\left(S_{r}\right)$. Now, assume that $\Delta$ is $\left(S_{2}\right)$. Let $G$ be a facet of $\Delta_{0} \cap\langle F\rangle$. By the same argument as in the proof of Theorem 3.2, we conclude that $\operatorname{lk}_{\Delta}(G)$ is not connected and so, by Corollary $2.4, \operatorname{dim}\left(\mathrm{lk}_{\Delta}(G)\right)=0$, which means that $\Delta_{0} \cap\langle F\rangle$ is a pure $(d-2)$-dimensional simplical complex. Therefore, the geometric realization of $\Delta_{0} \cap\langle F\rangle$ is homeomorphic either to the $(d-2)$-ball or to the $(d-2)$-sphere. In both cases, for every face $\sigma$ of $\Delta_{0} \cap\langle F\rangle$ and for every $i \leq \operatorname{dim}\left(\mathrm{lk}_{\Delta_{0} \cap\langle F\rangle}(\sigma)\right)$, we have $\widetilde{H}_{i}\left(\operatorname{lk}_{\Delta_{0} \cap\langle F\rangle}(\sigma) ; \mathbb{K}\right)=0$. Now assume that $\tau$ is an arbitrary face of $\Delta$. We now claim that for every $-1 \leq i \leq r-2$ with $|\tau| \leq d-i-2$, we have $\widetilde{H}_{i}\left(\mathrm{lk}_{\Delta}(\tau) ; \mathbb{K}\right)=0$. We prove the claim by using Theorem 2.2. There are two possibilities: either $\tau$ is not a face of $\Delta_{0} \cap\langle F\rangle$ or $\tau$ is a face of $\Delta_{0} \cap\langle F\rangle$.

Case 1. Assume that $\tau$ is not a face of $\Delta_{0} \cap F$. Then either $\mathrm{lk}_{\Delta}(\tau)=\mathrm{lk}_{\Delta_{0}}(\tau)$ or $\mathrm{lk}_{\Delta}(\tau)=\mathrm{lk}_{\langle F\rangle}(\tau)$. Since $\Delta_{0}$ is $\left(S_{r}\right)$ and the simplex $\langle F\rangle$ is Cohen-Macaulay, in both cases, for every $-1 \leq i \leq r-2$ with $|\tau| \leq d-i-2$, we have $\widetilde{H}_{i}\left(\mathrm{lk}_{\Delta}(\tau) ; \mathbb{K}\right)=0$.

Case 2. Assume that $\tau$ is a face of $\Delta_{0} \cap\langle F\rangle$. Then $\mathrm{lk}_{\Delta}(\tau)=$ $\mathrm{lk}_{\Delta_{0}}(\tau) \cup \mathrm{lk}_{\langle F\rangle}(\tau)$. By the same argument as Case 1, for every 
$i \leq d-2-|\tau|$, we have $\widetilde{H}_{i}\left(\mathrm{lk}_{\Delta_{0} \cap\langle F\rangle}(\tau) ; \mathbb{K}\right)=0$.

Therefore, the claim holds true. Now, applying the Mayer-Vietoris sequence completes the proof.

4. Edge ideals. Let $\mathbb{K}$ be a field. To any finite simple graph $G$ with vertex set $V(G)=\left\{v_{1}, \ldots, v_{n}\right\}$ and edge set $E(G)$, one associates an ideal $I(G) \subset \mathbb{K}\left[x_{1}, \ldots, x_{n}\right]$ generated by all monomials $x_{i} x_{j}$ such that $\left\{v_{i}, v_{j}\right\} \in E(G)$. The ideal $I(G)$ and the quotient ring $\mathbb{K}\left[x_{1}, \ldots, x_{n}\right] / I(G)$ are called the edge ideal of $G$ over $\mathbb{K}$ and the edge ring of $G$ over $\mathbb{K}$, respectively. The edge ideal $I(G)$ of $G$ was first introduced by Villarreal [42]. Subsequently, many people, including $[1,8,9,17,19,34,35,41]$, have been working on a program to build a dictionary between the algebraic properties of $I(G)$ and the combinatorial structure of $G$. The independence simplicial complex of $G$ is defined by

$$
\Delta_{G}=\{A \subseteq V(G) \mid A \text { is an independent set in } G\} .
$$

We recall that $A \subseteq V(G)$ is an independent set in $G$ if none of its elements are adjacent. Note that $\Delta_{G}$ is precisely the simplicial complex with the Stanley-Reisner ideal $I(G)$.

Let $G$ be a graph with vertex set $V(G)$ and edge set $E(G)$. A subset $C \subseteq V(G)$ is a minimal vertex cover of $G$ if, first, every edge of $G$ is incident with a vertex in $C$ and, second, there is no proper subset of $C$ with the first property. Note that $C$ is a minimal vertex cover if and only if $V(G) \backslash C$ is a maximal independent set, that is, a facet of $\Delta_{G}$. A graph $G$ is called unmixed if all minimal vertex covers of $G$ have the same number of elements, that is, $\Delta_{G}$ is pure. A graph is called chordal if every cycle of length at least four has a chord. We recall that a chord of a cycle is an edge which joins two vertices of the cycle but is not itself an edge of the cycle.

For a given field $\mathbb{K}$, a graph $G$ is said to be Cohen-Macaulay (respectively, $\left(S_{r}\right)$ ) over $\mathbb{K}$, if the edge ring $\mathbb{K}\left[x_{1}, \ldots, x_{n}\right] / I(G)$ of $G$ is Cohen-Macaulay (respectively, $\left(S_{r}\right)$ ) and it is called shellable, if $\Delta_{G}$ is shellable.

A satisfactory classification of all Cohen-Macaulay graphs over a field $\mathbb{K}$ has been standing open for some time. However, as pointed out 
in the introduction of the paper [19] by Herzog et al., this is equivalent to a classification of all Cohen-Macaulay simplicial complexes over $\mathbb{K}$ which is clearly a hard problem. Accordingly, it is natural to study special families of Cohen-Macaulay graphs. We recall that a graph $G$ on the vertex set $V(G)=\left\{v_{1}, \ldots, v_{n}\right\}$ is bipartite if there exists a partition $V(G)=U \cup W$ with $U \cap W=\varnothing$ such that each edge of $G$ is of the form $\left\{v_{i}, v_{j}\right\}$ with $v_{i} \in U$ and $v_{j} \in W$. If a graph $G$ is not bipartite, then $G$ contains a cycle of odd length. This result was found by the Hungarian mathematician, Dénes König (1884-1944), in 1916 (see [22]). His celebrated textbook Theorie der endlichen und unendlichen Graphen [23] appeared in 1936, and was the first book to present graph theory as a subject in its own right. Note that the converse of König 's result is also true, and so the bipartite graphs are characterized by the absence of cycles of odd length. For a Cohen-Macaulay bipartite graph $G$, Estrada and Villarreal [7] showed that $G \backslash\{\nu\}$ is Cohen-Macaulay for some vertex $\nu \in V(G)$. Herzog and Hibi gave a graph theoretical characterization of all bipartite Cohen-Macaulay graphs. In [12], it was shown that a bipartite graph is Cohen-Macaulay if and only if it is $\left(S_{2}\right)$. Now we state the results which provide a characterization of bipartite Cohen-Macaulay and $\left(S_{2}\right)$ graphs.

Theorem 4.1. ([15], Theorem 3.4), ([12], Theorem 1.3). Let $G$ be a bipartite graph with at least four vertices and with vertex partitions $U$ and $W$. Then the following conditions are equivalent:

(a) $G$ is unmixed and $U$ and $W$ can be labeled in such a way that there exists an order $U=F_{0}, \ldots, F_{n}=W$ of the facets of $\Delta_{G}$ where $F_{i}$ and $F_{i+1}$ intersect in codimension one for $0 \leq i \leq n-1$.

(b) $|U|=|W|$ and the vertices $U=\left\{x_{1}, \ldots, x_{n}\right\}$ and $W=$ $\left\{y_{1}, \ldots, y_{n}\right\}$ can be labeled in such a way that

(1) $\left\{x_{i}, y_{i}\right\}$ are edges for $1 \leq i \leq n$,

(2) if $\left\{x_{i}, y_{j}\right\}$ is an edge, then $i \leq j$, and

(3) if $\left\{x_{i}, y_{j}\right\}$ and $\left\{x_{j}, y_{k}\right\}$ are edges, then $\left\{x_{i}, y_{k}\right\}$ is also an edge. 
(c) G is a Cohen-Macaulay graph over a field.

(d) $G$ is an $\left(S_{2}\right)$ graph.

Note that this result is independent from the characteristic of the base field.

Remark 4.2. Theorem 4.1 reveals that, for bipartite graphs, CohenMacaulay and $\left(S_{2}\right)$ properties are equivalent. This raises the question whether there are other families of graphs for which these two properties are equivalent. In fact, one can show that a chordal graph is $\left(S_{2}\right)$ if and only if it is Cohen-Macaulay (see [12, Remark 1.5]). Also, it is shown that the cycle graph $C_{7}$ is the only cyclic graph which is $\left(S_{2}\right)$ but not Cohen-Macaulay (see [12, Proposition 1.6]).

Let $\Delta$ be a simplicial complex. A facet $F$ of $\Delta$ is called a leaf if there is a facet $G \neq F$ of $\Delta$, called a branch of $F$, in such a way that $H \cap F \subseteq G \cap F$ holds true for all facets $H$ of $\Delta$ with $H \neq F$. A quasi-forest simplicial complex is a simplicial complex $\Delta$, which enjoys an ordering $F_{1}, F_{2}, \ldots, F_{s}$ of the facets of $\Delta$, called a leaf order, in such a way that for every $j$ with $1 \leq j \leq s$, the facet $F_{j}$ is a leaf of the subcomplex $\left\langle F_{1}, \ldots, F_{j}\right\rangle$.

We close this section with the following proposition.

Proposition 4.3 ([10], Proposition 2.4). Let $\mathbb{K}$ be a field and $\Delta a$ $(d-1)$-dimensional quasi-forest simplicial complex. Then $\Delta$ is CohenMacaulay over $\mathbb{K}$ if and only if $\Delta$ satisfies Serre's condition $\left(S_{2}\right)$.

5. Sequentially Serre's condition. A nice generalization of CohenMacaulay modules is the notion of sequentially Cohen-Macaulay modules introduced first by Stanley [36]. This notion is, in fact, a nonpure generalization of the notion of Cohen-Macaulayness. Stanley introduced this notion in order to provide a ring theoretical complement to the theory of nonpure shellability [2]. Just as pure shellability implies Cohen-Macaulayness, nonpure shellability implies sequentially CohenMacaulayness. 
Definition 5.1. Let $M$ be a finitely generated $\mathbb{Z}$-graded module over a standard graded $\mathbb{K}$-algebra $R$, where $\mathbb{K}$ is a field. We say that $M$ is sequentially Cohen-Macaulay, if there exists a finite filtration $0=M_{0} \subset M_{1} \subset \cdots \subset M_{t}=M$ of $M$ by graded submodules $M_{i}$ satisfying the following two conditions:

(1) Every quotient $M_{i} / M_{i-1}$ is Cohen-Macaulay.

(2) $\operatorname{dim}\left(M_{1} / M_{0}\right)<\operatorname{dim}\left(M_{2} / M_{1}\right)<\cdots<\operatorname{dim}\left(M_{t} / M_{t-1}\right)$.

We say that a simplicial complex $\Delta$ is sequentially Cohen-Macaulay over a field $\mathbb{K}$, if the Stanley-Reisner ring $\mathbb{K}[\Delta]$ of $\Delta$ is sequentially Cohen-Macaulay.

As a generalization of Serre's condition and sequentially CohenMacaulay modules the authors of [11] have defined the notion of sequentially $\left(S_{r}\right)$ modules as follows:

Definition 5.2. Let $M$ be a finitely generated $\mathbb{Z}$-graded module over a standard graded $\mathbb{K}$-algebra $R$, where $\mathbb{K}$ is a field. We say that $M$ is sequentially $\left(S_{r}\right)$, if there exists a finite filtration $0=M_{0} \subset M_{1} \subset$ $\cdots \subset M_{t}=M$ of $M$ by graded submodules $M_{i}$ satisfying the following two conditions:

(1) Every quotient $M_{i} / M_{i-1}$ is $\left(S_{r}\right)$.

(2) $\operatorname{dim}\left(M_{1} / M_{0}\right)<\operatorname{dim}\left(M_{2} / M_{1}\right)<\cdots<\operatorname{dim}\left(M_{t} / M_{t-1}\right)$.

We say that a simplicial complex $\Delta$ is sequentially $\left(S_{r}\right)$ over a field $\mathbb{K}$, if the Stanley-Reisner ring $\mathbb{K}[\Delta]$ of $\Delta$ is sequentially $\left(S_{r}\right)$.

As analogues of a result by Duval ([4, Theorem 3.3]) for characterizing sequentially Cohen-Macaulay simplicial complexes, the authors of [11] have proved the following result.

Theorem 5.3. Let $\mathbb{K}$ be a field and $\Delta a(d-1)$-dimensional simplicial complex. Then $\Delta$ is sequentially $\left(S_{r}\right)$ over $\mathbb{K}$ if and only if for all $-1 \leq i \leq d-1$ its pure $i$-skeleton $\Delta^{[i]}$ is $\left(S_{r}\right)$ over $\mathbb{K}$.

The above theorem has the following useful corollary. 
Corollary 5.4. Let $\mathbb{K}$ be a field and $\Delta$ a simplicial complex. Then $\Delta$ is $\left(S_{r}\right)$ over $\mathbb{K}$ if and only if it is pure and sequentially $\left(S_{r}\right)$ over $\mathbb{K}$.

The following characterization of sequentially $\left(S_{r}\right)$ simplicial complexes over a field also has been proved (see [11, Theorem 2.9]).

Theorem 5.5. Let $\Delta$ be a simplicial complex with vertex set $V$. Then $\Delta$ is sequentially $\left(S_{2}\right)$ if and only if the following two conditions hold:

(1) $\Delta^{[i]}$ is connected for all $i \geq 1$.

(2) For all $x \in V, \mathrm{lk}_{\Delta}(x)$ is sequentially $\left(S_{2}\right)$.

Consider $R=\mathbb{K}\left[x_{1}, \ldots, x_{n}\right]$ with $\operatorname{deg}\left(x_{i}\right)=1$ for all $i$. If $I$ is a homogenous ideal of $R$ and $r \geq 1$, then $I$ is said to have linear resolution if, for some integer $d, \beta_{i, i+t}(I)=0$ for all $i$ and every $t \neq d$. More generally, $I$ is said to be linear in the first $r$ steps if, for some integer $d, \beta_{i, i+t}(I)=0$ for all $0 \leq i<r$ and $t \neq d$. We write $I_{\langle j\rangle}$ for the ideal generated by all homogenous polynomials of degree $j$ belonging to $I$. We say that a homogenous ideal $I \subset R$ is componentwise linear if $I_{\langle j\rangle}$ has a linear resolution for all $j$. The ideal $I$ is said to be componentwise linear in the first $r$ steps if, for all $j \geq 0, I_{\langle j\rangle}$ is linear in the first $r$ steps. A simplicial complex $\Delta$ on $[n]$ is said to be linear in the first $r$ steps, componentwise linear and componentwise linear in the first $r$ steps, if $I_{\Delta}$ satisfies either of these properties, respectively.

For a graph $G$ with vertex set $V(G)=\left\{v_{1}, \ldots, v_{n}\right\}$ and edge set $E(G)$, the cover ideal of $G$ is defined by

$$
J_{G}=\bigcap_{\left\{v_{i}, v_{j}\right\} \in E(G)}\left\langle x_{i}, x_{j}\right\rangle .
$$

For instance, unmixed squarefree monomial ideals of codimension two are just cover ideals of graphs. The name cover ideal comes from the fact that $J_{G}$ is generated by squarefree monomials $x_{i_{1}}, \ldots, x_{i_{r}}$ with $\left\{v_{i_{1}}, \ldots, v_{i_{r}}\right\}$ a minimal vertex cover of $G$. One can easily see that $J_{G}=I(G)^{\vee}$. We recall that, for a graph $G=(V(G), E(G))$, its complementary graph $\bar{G}$ is a graph with $V(\bar{G})=V(G)$, and $E(\bar{G})$ consists of those 2-element subsets $\left\{v_{i}, v_{j}\right\}$ of $V(G)$ for which $\left\{v_{i}, v_{j}\right\} \notin E(G)$. 
A major result of Eagon and Reiner states that a simplicial complex is Cohen-Macaulay if and only if the Stanley-Reisner ideal of its Alexander dual has a linear resolution (see [5, Theorem 3]). Fröberg has proved that, for every graph $G$, the edge ideal $I(G)$ of $G$ has a linear resolution if and only if the complementary graph $\bar{G}$ of $G$ is chordal. Combining these two results, we get the following theorem.

Theorem 5.6. Let $G$ be a graph with $n$ vertices, $R=\mathbb{K}\left[x_{1}, \ldots, x_{n}\right]$ the polynomial ring in $n$ variables over a field $\mathbb{K}$, and let $J_{G} \subseteq R$ be the cover ideal of $G$. Then $R / J_{G}$ is Cohen-Macaulay if and only if the complementary graph $\bar{G}$ of $G$ is chordal.

The following result due to Terai and Yanagawa is a generalization of Eagon and Reiner's result (see [44, Corollary 3.7]).

Theorem 5.7. A simplicial complex is $\left(S_{r}\right)$ if and only if the minimal free resolution of its Alexander dual is linear in the first $r$ steps.

Eisenbud, Green, Hulek and Popescu have generalized the result of Fröberg. They have proved that, for every graph $G$, the minimal free resolution of the edge ideal $I(G)$ of $G$ is linear in the first $r$ steps if and only if every minimal cycle in $\bar{G}$ has length at least $r+3$ (see [6, Theorem 2.1]). Combining this result with Theorem 5.7 implies the following generalization of Theorem 5.6.

Theorem 5.8. Let $G$ be a graph with $n$ vertices, $R=\mathbb{K}\left[x_{1}, \ldots, x_{n}\right]$ the polynomial ring in $n$ variables over a field $\mathbb{K}$, and let $J_{G} \subseteq R$ be the cover ideal of $G$. Then $R / J_{G}$ is $\left(S_{r}\right)$ if and only if every minimal cycle in $\bar{G}$ has length at least $r+3$.

Herzog, Hibi and Zheng have proved that, if $I$ is a monomial ideal generated in degree two, then $I$ has linear resolution if and only if every power of $I$ has linear resolution (see [18, Theorem 3.2]). Now it is natural to ask the following question.

Question 5.9. Let I be a monomial ideal generated in degree two, such that I is linear in the first $r$ steps. Is it true that every power of I satisfies the same property? 
Also, Herzog and Hibi have generalized the result of Eagon and Reiner in another direction. They have proved that a simplicial complex is sequentially Cohen-Macaulay if and only if the minimal free resolution of the Stanley-Reisner ideal of its Alexander dual is componentwise linear (see [14, Theorem 2.1]).

In [11], the authors have generalized the above-mentioned result of Herzog and Hibi as follows:

Theorem 5.10 ([11], Theorem 3.2). Let $\Delta$ be a simplicial complex on $[n]$. Then the Stanley-Reisner ideal of $\Delta$ is componentwise linear in the first $r$ steps if and only if $\Delta^{\vee}$, the Alexander dual of $\Delta$, is sequentially $\left(S_{r}\right)$.

Sequentially Cohen-Macaulay cycles have been characterized by Francisco and Van Tuyl (see [9, Proposition 4.1]). These cycles are just $C_{3}$ and $C_{5}$. Woodroofe has given a more geometric proof for this result (see [43, Theorem 10]). In [12, Proposition 1.6], it is shown that the only $\left(S_{2}\right)$ cycles are $C_{3}, C_{5}$ and $C_{7}$. In [11], the authors have generalized this result, and they have proved that the odd cycles are sequentially $\left(S_{2}\right)$ which are the only sequentially $\left(S_{2}\right)$ cycles. Also, they have proved that $C_{3}$ and $C_{5}$ are the only sequentially $\left(S_{3}\right)$ cycles.

Definition 5.11. Let $\Delta$ be a simplicial complex on the vertex set $V=\left\{x_{1}, \ldots, x_{n}\right\}$. Then we say that $\Delta$ is vertex decomposable if either

(1) $\Delta$ is a simplex, or

(2) there exists $x \in V$ such that $\operatorname{del}_{\Delta}(x)$ and $\operatorname{lk}_{\Delta}(x)$ are vertex decomposable and every facet of $\operatorname{del}_{\Delta}(x)$ is a facet of $\Delta$.

A graph $G$ is called vertex decomposable if $\Delta_{G}$ is vertex decomposable.

Now, we may state the following results.

Theorem 5.12. ([11], Theorem 4.5), ([41], Theorem 2.10). Let $G$ be a bipartite graph. Then the following conditions are equivalent:

(a) $G$ is vertex decomposable. 
(b) $G$ is shellable.

(c) $G$ is sequentially Cohen-Macaulay.

(d) $G$ is sequentially $\left(S_{2}\right)$.

Theorem 5.13 ([43], Theorem 1). Let $G$ be a graph with no chordless cycles of length other than 3 or 5 . Then $G$ is vertex decomposable and it is, hence, shellable, sequentially Cohen-Macaulay and sequentially $\left(S_{2}\right)$.

We are now in a position to state and prove the following generalization of Theorem 3.2 for nonpure case.

Theorem 5.14. Let $\mathbb{K}$ be a field and $\Delta$ a simplicial complex. Then the following conditions are equivalent:

(a) There exists an ordering of facets of $\Delta$, say $F_{0}, \ldots, F_{m}$, such that, for every $0 \leq i \leq m$, the simplicial complex $\left\langle F_{0}, \ldots, F_{i}\right\rangle$ is sequentially Cohen-Macaulay over $\mathbb{K}$.

(b) There exists an ordering of facets of $\Delta$, say $F_{0}, \ldots, F_{m}$, such that, for every $0 \leq i \leq m$, the simplicial complex $\left\langle F_{0}, \ldots, F_{i}\right\rangle$ is sequentially $\left(S_{2}\right)$.

(c) $\Delta$ is shellable.

Proof. The implications (c) $\Rightarrow$ (a) and (a) $\Rightarrow$ (b) are trivial. (Note that as an extension of Theorem 3.1, it was proved by Stanley [36] that every shellable simplicial complex is sequentially Cohen-Macaulay.) We now prove that (b) implies (c).

Let $\Delta$ be a simplicial complex of dimension $d-1$. If $d=1$, then $\Delta$ is a zero-dimensional simplicial complex, and thus it is shellable. Hence, we assume that $d \geq 2$ and suppose that there exists an ordering of facets of $\Delta$, say $F_{0}, \ldots, F_{m}$, such that, for every $0 \leq i \leq m$, the simplicial complex $\left\langle F_{0}, \ldots, F_{i}\right\rangle$ is sequentially $\left(S_{2}\right)$. We prove that this ordering is, in fact, a shelling order, and this proves (c). For this, it is enough to prove that, for every $1 \leq i \leq m,\left\langle F_{0}, \ldots, F_{i-1}\right\rangle \cap\left\langle F_{i}\right\rangle$ is pure of dimension $\operatorname{dim} F_{i}-1$. Now suppose, on the contrary, that 
there exists $1 \leq i \leq m$ such that $\left\langle F_{0}, \ldots, F_{i-1}\right\rangle \cap\left\langle F_{i}\right\rangle$ is not pure of dimension $\operatorname{dim} F_{i}-1$. This implies that $\left\langle F_{0}, \ldots, F_{i-1}\right\rangle \cap\left\langle F_{i}\right\rangle$ has a facet, say $G$, with dimension at most $\operatorname{dim} F_{i}-2$. Let $\Delta_{i}:=\left\langle F_{0}, \ldots, F_{i}\right\rangle$ and $t=\operatorname{dim} F_{i}$. Similar to the proof of Theorem 3.2, one can show that $\mathrm{lk}_{\Delta_{i}[t]}(G)$ is not connected. Note that, by [11, Lemma 2.2, Theorem 2.6], $\operatorname{lk}_{\Delta_{i}[t]}(G)$ is $\left(S_{2}\right)$ and $\operatorname{dim}\left(\operatorname{lk}_{\Delta_{i}[t]}(G)\right) \geq 1$. Now Corollary 2.4 implies that $\operatorname{lk}_{\Delta_{i}[t]}(G)$ is connected, and this is a contradiction.

6. $f$ - and $h$-vectors. We now record the numerical data associated to a $(d-1)$-dimensional simplicial complex $\Delta$. Let $f_{i}$ denote the number of faces of $\Delta$ of dimension $i$. The sequence $f(\Delta)=\left(f_{0}, f_{1}, \ldots, f_{d-1}\right)$ is called the $f$-vector of $\Delta$. Terai and Yoshida have proved the following result.

Theorem 6.1 ([40], Theorem 2.1). Let $\Delta$ be a $(d-1)$-dimensional $\left(S_{r}\right)$ simplicial complex, and let $f(\Delta)=\left(f_{0}, f_{1}, \ldots, f_{d-1}\right)$ be its $f$-vector. If $f_{d-1} \geq\left(\begin{array}{c}f_{0} \\ d\end{array}\right)-3\left(f_{0}-d\right)+2$, then $\Delta$ is Cohen-Macaulay.

To find out more about $f(\Delta)$, we need to study the $h$-vector of $\Delta$. Although it is seemingly complicated, there often is an elegant way to record the face numbers. Letting $f_{-1}=1$, we define the $h$-vector $h(\Delta)=\left(h_{0}, h_{1}, \ldots, h_{d}\right)$ of $\Delta$ by the formula

$$
\sum_{i=0}^{d} f_{i-1}(t-1)^{d-i}=\sum_{i=0}^{d} h_{i} t^{d-i}
$$

One can easily check that

$$
f_{i-1}=\sum_{j=0}^{i}\left(\begin{array}{l}
d-j \\
i-j
\end{array}\right) h_{j}
$$

and

$$
h_{i}=\sum_{j=0}^{i}(-1)^{i-j}\left(\begin{array}{l}
d-j \\
i-j
\end{array}\right) f_{j-1} .
$$

It is well known that, if $h(\Delta)=\left(h_{0}, h_{1}, \ldots, h_{d}\right)$ is the $h$-vector of $\Delta$ 
and $H_{\mathbb{K}[\Delta]}(t)$ is the Hilbert series of $\mathbb{K}[\Delta]$, then we have

$$
H_{\mathbb{K}[\Delta]}(t)=\frac{\sum_{i=0}^{d} h_{i} t^{i}}{(1-t)^{d}},
$$

where $d=\operatorname{dim} \Delta+1$ is the Krull dimension of $\mathbb{K}[\Delta]$.

The study of $h$-vectors of simplicial complexes has long been a topic of interest both in combinatorics and combinatorial commutative algebra. Let $\Delta$ be a $(d-1)$-dimensional simplicial complex, and let $h(\Delta)=\left(h_{0}, h_{1}, \ldots, h_{d}\right)$ be its $h$-vector. A classical result of Stanley guarantees that, if $\Delta$ is Cohen-Macaulay, then $h_{i}$ is nonnegative for every $i$ with $0 \leq i \leq d$ (see [36, Page 59, Theorem 3.3]).

Murai and Terai [28] have proved that if $\Delta$ is a $(d-1)$-dimensional $\left(S_{r}\right)$ simplicial complex and $h(\Delta)=\left(h_{0}, h_{1}, \ldots, h_{d}\right)$ is its $h$-vector, then the following two conditions hold:

$\circ\left(h_{0}, h_{1}, \ldots, h_{r}\right)$ is an $M$-vector, and

$\circ h_{r}+h_{r+1}+\cdots+h_{d}$ is nonnegative.

Note that, in the above, a sequence of integers $\mathfrak{h}=\left(h_{0}, h_{1}, \ldots, h_{r}\right)$ is called an $M$-vector provided there exists a Cohen-Macaulay $(r-1)$ dimensional simplicial complex $\Gamma$ with $h(\Gamma)=\mathfrak{h}$. Now, it is natural to ask whether the above two conditions are sufficient for a simplicial complex to be $\left(S_{r}\right)$. This is not the case, even for the independence simplicial complex of cycle graphs, as $C_{9}$ shows. In [10], the authors have extended the results of Murai and Terai by giving $r$ extra necessary conditions. More precisely, they have proved the following result.

Theorem 6.2. [28], ([10], Theorem 2.1). Let $\Delta$ be a (d-1)-dimensional $\left(S_{r}\right)$ simplicial complex, and let $h(\Delta)=\left(h_{0}, h_{1}, \ldots, h_{d}\right)$ be its $h$ vector. Then the following conditions hold:

(1) $\left(h_{0}, h_{1}, \ldots, h_{r}\right)$ is an $M$-vector, and

(2) $\left(\begin{array}{l}i \\ i\end{array}\right) h_{r}+\left(\begin{array}{c}i+1 \\ i\end{array}\right) h_{r+1}+\cdots+\left(\begin{array}{c}i+d-r \\ i\end{array}\right) h_{d}$ is nonnegative for every $i$ with $0 \leq i \leq r \leq d$. 
One can easily check that conditions (1) and (2) stated in Theorem 6.2 are sufficient for the independence simplicial complex of cycle graphs to be $\left(S_{r}\right)$. Now, it is natural to ask whether the converse of Theorem 6.2 is true in general.

Question 6.3 ([10], Question 2.6). Let $d$ and $r$ be integers with $d \geq r \geq 2$, and let $\mathfrak{h}=\left(h_{0}, h_{1}, \ldots, h_{d}\right)$ be the $h$-vector of a simplicial complex in such a way that the following conditions hold:

(1) $\left(h_{0}, h_{1}, \ldots, h_{r}\right)$ is an $M$-vector, and

(2) $\left(\begin{array}{c}i \\ i\end{array}\right) h_{r}+\left(\begin{array}{c}i+1 \\ i\end{array}\right) h_{r+1}+\cdots+\left(\begin{array}{c}i+d-r \\ i\end{array}\right) h_{d}$ is nonnegative for every $i$ with $0 \leq i \leq r \leq d$.

Does there exist a $(d-1)$-dimensional $\left(S_{r}\right)$ simplicial complex $\Delta$ with $h(\Delta)=\mathfrak{h}$ ?

We close this section by stating a result which has been proved by Murai and Terai.

Theorem 6.4 ([28], Theorem 1.2). Let $\Delta$ be a $(d-1)$-dimensional $\left(S_{r}\right)$ simplicial complex, and let $h(\Delta)=\left(h_{0}, h_{1}, \ldots, h_{d}\right)$ be its $h$-vector. If $h_{t}=0$ for some $t \leq r$, then $h_{k}=0$ for all $k \geq t$ and $\Delta$ is CohenMacaulay.

7. Power and symbolic power of ideals. Let $R=\mathbb{K}\left[x_{1}, \ldots, x_{n}\right]$ be the polynomial ring in $n$ variables over a field $\mathbb{K}$, and let $\Delta$ be a simplicial complex on $[n]$. We say that $\Delta$ is complete intersection over $\mathbb{K}$, if the Stanley-Reisner ring $\mathbb{K}[\Delta]$ of $\Delta$ is a complete intersection ring. Combinatorially, this means that the minimal nonfaces of $\Delta$ are disjoint.

A simplicial complex $\Delta$ is called matroid if it is a collection of subsets of a finite set, called independent sets, with the following three properties:

(1) The empty set is independent.

(2) Every subset of an independent set is independent. 
(3) If $F$ and $G$ are two independent sets and $F$ has more elements than $G$, then there exists an element in $F$ which is not in $G$ that when added to $G$ still gives an independent set.

Let $I$ be a squarefree monomial ideal in the polynomial $\operatorname{ring} R=$ $\mathbb{K}\left[x_{1}, \ldots, x_{n}\right]$ over a field $\mathbb{K}$, and suppose that $I$ has the primary decomposition

$$
I=\mathfrak{p}_{1} \cap \cdots \cap \mathfrak{p}_{r},
$$

where every $\mathfrak{p}_{i}$ is an ideal of $R$ generated by a subset of the variables of $R$. Let $k$ be a positive integer. The $k$ th symbolic power of $I$, denoted by $I^{(k)}$, is defined to be

$$
I^{(k)}=\mathfrak{p}_{1}^{k} \cap \cdots \cap \mathfrak{p}_{r}^{k} .
$$

In [31], the authors have examined the Cohen-Macaulay property of the symbolic powers of edge ideals of graphs, and they have proved the following result.

Theorem 7.1 ([31], Theorem 3.6). Let $R=\mathbb{K}\left[x_{1}, \ldots, x_{n}\right]$ be the polynomial ring in $n$ variables over a field $\mathbb{K}$, and let $I(G)$ be the edge ideal of a graph $G$. Then the following conditions are equivalent:

(a) $R / I(G)^{(m)}$ is Cohen-Macaulay for every $m \geq 1$.

(b) $R / I(G)^{(m)}$ is Cohen-Macaulay for every $m \geq 3$.

(c) $R / I(G)^{(m)}$ satisfies the Serre's condition $\left(S_{2}\right)$ for every $m \geq 3$.

(d) $R / I(G)^{(m)}$ satisfies the Serre's condition $\left(S_{2}\right)$ for some $m \geq 3$.

(e) $G$ is a disjoint union of finitely many complete graphs.

Terai and Trung have generalized Theorem 7.1, and they have proved the following two theorems. The second one is an analogue of the first one and is for ordinary powers of Stanley-Reisner ideals.

Theorem 7.2 ([39], Theorem 1.1). Let $R=\mathbb{K}\left[x_{1}, \ldots, x_{n}\right]$ be the polynomial ring in $n$ variables over a field $\mathbb{K}$, and let $\Delta$ be a simplicial 
complex on $[n]$ with $\operatorname{dim} \Delta \geq 2$. Then the following conditions are equivalent:

(a) $R / I_{\Delta}^{(m)}$ is Cohen-Macaulay for every $m \geq 1$.

(b) $R / I_{\Delta}^{(m)}$ is Cohen-Macaulay for every $m \geq 3$.

(c) $R / I_{\Delta}^{(m)}$ satisfies the Serre's condition $\left(S_{2}\right)$ for every $m \geq 3$.

(d) $R / I_{\Delta}^{(m)}$ satisfies the Serre's condition $\left(S_{2}\right)$ for some $m \geq 3$.

(e) $\Delta$ is a matroid.

Theorem 7.3 ([39], Theorem 1.2). Let $R=\mathbb{K}\left[x_{1}, \ldots, x_{n}\right]$ be the polynomial ring in $n$ variables over a field $\mathbb{K}$, and let $\Delta$ be a simplicial complex on $[n]$ with $\operatorname{dim} \Delta \geq 2$. Then the following conditions are equivalent:

(a) $R / I_{\Delta}^{m}$ is Cohen-Macaulay for every $m \geq 1$.

(b) $R / I_{\Delta}^{m}$ is Cohen-Macaulay for every $m \geq 3$.

(c) $R / I_{\Delta}^{m}$ satisfies the Serre's condition $\left(S_{2}\right)$ for every $m \geq 3$.

(d) $R / I_{\Delta}^{m}$ satisfies the Serre's condition $\left(S_{2}\right)$ for some $m \geq 3$.

(e) $\Delta$ is complete intersection.

Let $\Delta$ be a simplicial complex on the vertex set $V$. For every subset $U \subseteq V$, we denote by $\Delta_{U}$ the subcomplex of $\Delta$ whose facets are the facets of $\Delta$ with at least $|U|-1$ vertices in $U$. Minh and Trung [26] have characterized the Cohen-Macaulayness of $I_{\Delta}^{(2)}$ based on this notion.

Theorem 7.4 ([26], Theorem 2.1). Let $R=\mathbb{K}\left[x_{1}, \ldots, x_{n}\right]$ be the polynomial ring in $n$ variables over a field $\mathbb{K}$, and let $\Delta$ be a simplicial complex on the vertex set $V$. Then $R / I_{\Delta}^{(2)}$ is Cohen-Macaulay if and only if $\Delta$ is Cohen-Macaulay over $\mathbb{K}$ and $\Delta_{U}$ is Cohen-Macaulay over $\mathbb{K}$ for all subsets $U \subseteq V$ with $2 \leq|U| \leq \operatorname{dim} \Delta+1$. 
Let $G$ be a connected graph and $u, v$ two vertices of $G$. The distance between $u$ and $v$ is the minimal length of paths from $u$ to $v$. The diameter of $G$, denoted by $\operatorname{diam}(G)$, is the maximal distance between two vertices of $G$. We set $\operatorname{diam}(G)=\infty$ if $G$ is not a connected graph. Rinaldo, Terai and Yoshida have proved the following characterization of the $\left(S_{2}\right)$ property of the second symbolic power of Stanley-Reisner ideals.

Theorem 7.5 ([32], Theorem 0.1, Corollary 3.3). Let $R=\mathbb{K}\left[x_{1}, \ldots, x_{n}\right]$ be the polynomial ring in $n$ variables over a field $\mathbb{K}$, and let $\Delta$ be a pure simplicial complex. Then the following conditions are equivalent:

(a) $R / I_{\Delta}^{(2)}$ satisfies the Serre's condition $\left(S_{2}\right)$.

(b) $\operatorname{diam}\left(\left(\mathrm{lk}_{\Delta}(F)\right)^{(1)}\right) \leq 2$ for every face $F \in \Delta$ with $\operatorname{dim}\left(\mathrm{lk}_{\Delta}(F)\right) \geq$ 1.

Also, the equivalent conditions above are satisfied, provided that $R / I_{\Delta}^{2}$ is Cohen-Macaulay over $\mathbb{K}$.

8. Radical of ideals. There are simple examples of Cohen-Macaulay ideals whose radical is not Cohen-Macaulay. The first such example is probably due to Hartshorne [13]. By using either CoCoA or another computer algebra software, many other examples, even in characteristic zero, can be constructed. The following example due to Conca was computed using CoCoA.

Example 8.1. Let $\mathbb{K}$ be field, $R=\mathbb{K}\left[x_{1}, x_{2}, x_{3}, x_{4}, x_{5}\right]$, and let

$$
I=\left\langle x_{2}^{2}-x_{4} x_{5}, x_{1} x_{3}-x_{3} x_{4}, x_{3} x_{4}-x_{1} x_{5}\right\rangle \subset R .
$$

Then $R / I$ is a two dimensional Cohen-Macaulay ring, while $\sqrt{I}=\left\langle x_{1} x_{3}-x_{1} x_{5}, x_{3} x_{4}-x_{1} x_{5}, x_{2}^{2}-x_{4} x_{5}, x_{1}^{2} x_{2}-x_{1} x_{2} x_{4}, x_{2} x_{3}^{2}-x_{2} x_{3} x_{5}\right\rangle$, and so $S=R / \sqrt{I}$ is not Cohen-Macaulay. Indeed, the depth of $S$ is equal to 1.

On the other hand, it is well known that the Cohen-Macaulay property of a monomial ideal is inherited by its radical (see $[\mathbf{3 7}, \mathbf{2 0}]$ ). 
The reason is that the radical of a monomial ideal is essentially obtained by polarization and localization. By the same technique, one can prove that the $\left(S_{r}\right)$ property of monomial ideals is also inherited by its radical.

Herzog, Terai and Takayama have proved that the sequentially Cohen-Macaulay property of a monomial ideal is inherited by its radical (see [20, Theorem 2.6]). Now, it is natural to ask the following question.

Question 8.2. Let $R=\mathbb{K}\left[x_{1}, \ldots, x_{n}\right]$ be the polynomial ring in $n$ variables over a field $\mathbb{K}$, and let $I$ be a monomial ideal of $I$ such that $R / I$ is sequentially $\left(S_{2}\right)$. Is $R / \sqrt{I}$ sequentially $\left(S_{2}\right)$ too?

Let $R=\mathbb{K}\left[x_{1}, \ldots, x_{n}\right]$ be the polynomial ring in $n$ variables over a field $\mathbb{K}$, and let $I$ be a monomial ideal of $R$. It is proved that if $R / I$ is Cohen-Macaulay, then $R / \sqrt{I}$ is also Cohen-Macaulay (see [20, 38]). As a generalization of this result, we state and prove a similar result for Serre's condition. The technique of the proof is based on polarization, and so we recall this notion for the convenience of the reader.

Polarization is a deformation that assigns to an arbitrary monomial ideal a squarefree monomial ideal in a new set of variables. Let $I$ be a monomial ideal of $R=\mathbb{K}\left[x_{1}, \ldots, x_{n}\right]$ with minimal generators $u_{1}, \ldots, u_{m}$, where $u_{i}=\prod_{j=1}^{n} x_{j}^{a_{i j}}, 1 \leq i \leq m$. For every $j$ with $1 \leq j \leq n$, let $a_{j}=\max \left\{a_{i j} \mid 1 \leq i \leq m\right\}$, and suppose that

$$
T=\mathbb{K}\left[x_{11}, x_{12}, \ldots, x_{1 a_{1}}, x_{21}, x_{22}, \ldots, x_{2 a_{2}}, \ldots, x_{n 1}, x_{n 2}, \ldots, x_{n a_{n}}\right]
$$

is the polynomial ring over the field $\mathbb{K}$. Let $J$ be the squarefree monomial ideal of $T$ with minimal generators $v_{1}, \ldots, v_{m}$, where $v_{i}=$ $\prod_{j=1}^{n} \prod_{k=1}^{a_{i j}} x_{j k}, 1 \leq i \leq m$. The monomial $v_{i}$ is called the polarization of $u_{i}$, and the ideal $J$ is called the polarization of $I$.

Theorem 8.3. Let $R=\mathbb{K}\left[x_{1}, \ldots, x_{n}\right]$ be the polynomial ring in $n$ variables over a field $\mathbb{K}$, and let $I$ be a monomial ideal of $R$. If $R / I$ is an $\left(S_{r}\right)$ ring, then $R / \sqrt{I}$ is also an $\left(S_{r}\right)$ ring.

Proof. We prove the theorem by using the polarization trick. Let $T / J$ be the polarization of $R / I$, where $T=\mathbb{K}\left[x_{1}, \ldots, x_{n}, Y\right]$ is the new polynomial ring over $\mathbb{K}$ with $Y$ as the set of new variables. It is 
known that polarization preserves the $\left(S_{r}\right)$ property (see [28, Proof of Theorem 4.1]) and, therefore, since $R / I$ is $\left(S_{r}\right), T / J$ is also $\left(S_{r}\right)$. We now let $W$ be the multiplicatively closed subset $\mathbb{K}[Y] \backslash\{0\}$ in $T=R[Y]$ and consider $F=\mathbb{K}(Y)$. Then the localization of $T / J$ at $W$ is isomorphic to $F\left[x_{1}, \ldots, x_{n}\right] / \sqrt{I}$. Since $T / J$ is $\left(S_{r}\right)$ and localization preserves the Serre's condition, we conclude that

$$
F\left[x_{1}, \ldots, x_{n}\right] / \sqrt{I} \cong\left(\mathbb{K}\left[x_{1}, \ldots, x_{n}\right] / \sqrt{I}\right) \otimes_{\mathbb{K}} F
$$

is $\left(S_{r}\right)$. Now [3, Exercise 2.1.24] implies that

$$
\mathbb{K}\left[x_{1}, \ldots, x_{n}\right] / \sqrt{I}=R / \sqrt{I}
$$

is an $\left(S_{r}\right)$ ring, as required.

In order to state the next result we need the definition of the size of a monomial ideal: let $R=\mathbb{K}\left[x_{1}, \ldots, x_{n}\right]$ be the polynomial ring in $n$ variables over a field $\mathbb{K}$, and let $I$ be a monomial ideal of $R$. Lyubeznik [24] has defined the size of $I$, denoted by $\operatorname{size}(I)$, as follows. Let $I=\bigcap_{j=1}^{r} Q_{j}$ be an irredundant primary decomposition of $I$, where the $Q_{i}$ 's are monomial ideals. Let $h$ be the height of $\sum_{j=1}^{r} Q_{j}$, and denote by $v$ the minimum number $t$ such that there exist $j_{1}, \ldots, j_{t}$ with

$$
\sqrt{\sum_{j=1}^{r} Q_{j}}=\sqrt{\sum_{i=1}^{t} Q_{j_{i}}} .
$$

Then $\operatorname{size}(I)=v+(n-h)-1$.

We now close this section by stating and proving the following theorem. Here, for a subset $F \subseteq[n], P_{F}$ denotes the prime ideal generated by the $x_{i}$ 's with $i \in F$.

Theorem 8.4. Let I be a squarefree monomial ideal of $R=\mathbb{K}\left[x_{1}, \ldots, x_{n}\right]$, where $\mathbb{K}$ is a field and write $I=\bigcap_{i=1}^{r} P_{F_{i}}$, where the sets $F_{i} \subseteq[n]$ are pairwise distinct and all have the same cardinality c. For every $1 \leq i \leq r$ and $1 \leq j \leq c$, choose integers $a_{i j} \geq 1$ and set $Q_{F_{i}}=\left\langle x^{a_{i j}} \mid j \in F_{i}\right\rangle$. Then the following conditions are equivalent:

(a) For every choice of the integers $a_{i j}$, the ideal $I=\bigcap_{i=1}^{r} Q_{F_{i}}$ is Cohen-Macaulay. 
(b) For every subset $A \subseteq[r]$, the ideal $I_{A}=\bigcap_{i \in A} P_{F_{i}}$ is CohenMacaulay.

(c) For every choice of the integers $a_{i j}$, the ideal $I=\bigcap_{i=1}^{r} Q_{F_{i}}$ is $\left(S_{r}\right)$.

(d) For every subset $A \subseteq[r]$, the ideal $I_{A}=\bigcap_{i \in A} P_{F_{i}}$ is $\left(S_{r}\right)$.

(e) For every $i \neq j$, we have $\operatorname{ht}\left(P_{F_{i}}+P_{F_{j}}\right)=c+1$.

(f) For every $r \geq 2$, we have either $\left|\bigcup_{i=1}^{r} F_{i}\right|=c+1$, or $\left|\bigcap_{i=1}^{r} F_{i}\right|=c-1$.

(g) After a suitable permutation of the elements of $[n]$, for every $1 \leq i \leq r$ we have either $F_{i}=\{1, \ldots, i-1, i+1, \ldots, c, c+1\}$, or $F_{i}=\{1, \ldots, c-1, c-1+i\}$.

(h) $\operatorname{size}(I)=\operatorname{dim}(S / I)$.

(i) For every monomial ideal $L$ such that $\operatorname{Ass}(L)=\operatorname{Ass}(I), S / L$ is $\left(S_{r}\right)$.

(j) For every monomial ideal $L$ such that $\operatorname{Ass}(L)=\operatorname{Ass}(I), S / L$ is Cohen-Macaulay.

Proof. The proof of equivalence of conditions (a), (b), (e)-(h) and (j) can be found in [20]. The implications (a) $\Rightarrow(\mathrm{c}),(\mathrm{j}) \Rightarrow(\mathrm{i})$ and (i) $\Rightarrow$ (c) are trivial. The proofs of the remaining cases are as follows:

(c) $\Rightarrow$ (d). Let $Q_{F_{i}}=\left\langle x_{j}^{2} \mid j \in F_{i}\right\rangle$ if $i \in A$, and $Q_{F_{i}}=P_{F_{i}}$ if $i \notin A$. By the assumption, $J=\bigcap_{i=1}^{r} Q_{F_{i}}$ is $\left(S_{r}\right)$, and so the complete polarization $J^{p}$ of $J$ is also $\left(S_{r}\right)$. We have $J^{p}=\bigcap_{i=1}^{r} Q_{F_{i}}^{p}$, where $Q_{F_{i}}^{p}=\left\langle x_{j} y_{j} \mid j \in F_{i}\right\rangle$ if $i \in A$, and $Q_{F_{i}}^{p}=P_{F_{i}}$ if $i \notin A$. Let $N$ be the multiplicatively closed subset generated by all variables $x_{i}$. Then $J_{N}^{p}$ is $\left(S_{r}\right)$. But $J_{N}^{p}=\bigcap_{i \in A}\left\langle y_{j} \mid j \in F_{i}\right\rangle$. This shows that $I_{A}=\bigcap_{i \in A} P_{F_{i}}$ is $\left(S_{r}\right)$, as required.

(d) $\Rightarrow$ (e). Suppose, on the contrary, that there exists $i \neq j$ with $\operatorname{ht}\left(P_{F_{i}}+P_{F_{j}}\right) \geq c+2$. Let $\Delta$ be a simplicial complex such that $\mathbb{K}[\Delta]$ is isomorphic to $\mathbb{K}\left[F_{i} \cup F_{j}\right] /\left(P_{F_{i}} \cap P_{F_{j}}\right)$. Then the facets of $\Delta$ are just $F_{j} \backslash F_{i}$ and $F_{i} \backslash F_{j}$. Therefore, $\Delta$ is not connected. Since ht $\left(P_{F_{i}}+P_{F_{j}}\right) \geq c+2$, the dimension of $\Delta$ is at least two, and so $\Delta$ is not $\left(S_{r}\right)$. This contradicts (d).

9. Algebraic shifting. Algebraic shifting is a procedure that associates for every simplicial complex $\Delta$ a new simplicial complex $\Gamma(\Delta)$, called a shifted complex of $\Delta$, with the same $h$-vector as $\Delta$ and a nice combinatorial structure. Additionally, algebraic shifting preserves 
many algebraic and topological properties of the original complex, including Cohen-Macaulayness: a simplicial complex $\Delta$ is CohenMacaulay if and only if $\Gamma(\Delta)$ is Cohen-Macaulay, which, in turn, holds if and only if $\Gamma(\Delta)$ is pure (see [21]). The algebraic shifting operator was introduced by Kalai over 25 years ago, with applications mainly in the theory of $f$-vectors. Since then, connections and applications of this operator to other areas of mathematics, like algebraic topology and combinatorics, have been found by different researchers. For more information, we refer the reader to Kalai's recent survey [21].

In [29], the authors have proved the following result which is a partial generalization of Kalai's result.

Proposition 9.1. Let $\Delta$ be a simplicial complex, and let $\Gamma(\Delta)$ be its algebraic shifting. If $\Delta$ is $\left(S_{r}\right)$, then $\Gamma(\Delta)$ has no facet of dimension less than $r-1$.

Note that the converse of Proposition 9.1 is not true in general. In order to see this, let $\Delta$ be an $\left(S_{r}\right)$ simplicial complex which is not Cohen-Macaulay. Then $\Gamma(\Delta)$ is not pure while, by Proposition 9.1, $\Gamma(\Delta)$ has no facet of dimension less than $r-1$. Therefore, $\Gamma(\Gamma(\Delta))=$ $\Gamma(\Delta)$ has no facet of dimension less than $r-1$ and so is not $\left(S_{r}\right)$. Therefore, it is natural to ask the following question.

Question 9.2. What is the characterization of $\left(S_{r}\right)$ simplicial complexes via algebraic shifting?

Let $\Delta$ be a simplicial complex. The degree of a face $F \in \Delta$, denoted by $\operatorname{deg} F$, is defined to be $\operatorname{deg} F=\max \{|G| \mid F \subseteq G, G \in \Delta\}$. For every $i$ and $j$ with $0 \leq j \leq i \leq d$, let $f_{i, j}$ denote the number of faces of $\Delta$ of degree $i$ and dimension $j-1$, and consider

$$
h_{i, j}=\sum_{k=0}^{j}(-1)^{j-k}\left(\begin{array}{c}
i-k \\
j-k
\end{array}\right) f_{i, k} .
$$

Then the triangular integer arrays $\mathbf{f}=\left(f_{i, j}\right)_{0 \leq j \leq i \leq d}$ and $\mathbf{h}=\left(h_{i, j}\right)_{0 \leq j \leq i \leq d}$ are called the $f$-triangle and $h$-triangle of $\Delta$, respectively. 
Duval [4] has shown that algebraic shifting preserves the $h$-triangle of a simplicial complex $\Delta$, provided $\Delta$ is sequentially Cohen-Macaulay. The analogue of Duval's result is given in the following theorem.

Theorem 9.3 ([29], Theorem 3.2). Let $\Delta$ be a sequentially $\left(S_{r}\right)$ simplicial complex, and let $\Gamma(\Delta)$ be its algebraic shifting. Then for every $i$ and $j$ with $0 \leq j \leq i \leq r-1$, we have $h_{i, j}(\Delta)=h_{i, j}(\Gamma(\Delta))$.

Acknowledgments. The authors are grateful to the anonymous referee for making many constructive suggestions and for supplying some additional references.

\section{REFERENCES}

1. M. Barile, A note on the edge ideals of Ferrers graphs, arXiv : math/0606353v2.

2. A. Björner and M.L. Wachs, Shellable nonpure complexes and posets, I, Trans. Amer. Math. Soc. 348 (1996), 1299-1327.

3. W. Bruns and J. Herzog, Cohen-Macaulay rings, Cambridge Stud. Adv. Math. 39, Cambridge University Press, 1993.

4. A.M. Duval, Algebraic shifting and sequentially Cohen-Macaulay simplicial complexes, Electron. J. Combin. 3 (1996), Research Paper 21.

5. J.A. Eagon and V. Reiner, Resolutions of Stanley-Reisner rings and Alexander duality, J. Pure Appl. Alg. 130 (1998), 265-275.

6. D. Eisenbud, M. Green, K. Hulek and S. Popescu, Restricting linear syzygies: algebra and geometry, Compos. Math. 141 (2005), 1460-1478.

7. M. Estrada and R.H. Villarreal, Cohen-Macaulay bipartite graphs, Arch. Math. (Basel) 68 (1997), 124-128.

8. C.A. Francisco and H.T. Há, Whiskers and sequentially Cohen-Macaulay graphs, J. Combin. Theor. 115 (2008), 304-316.

9. C.A. Francisco and A. Van Tuyl, Sequentially Cohen-Macaulay edge ideals, Proc. Amer. Math. Soc. 135 (2007), 2327-2337.

10. A. Goodarzi, M.R. Pournaki, S.A. Seyed Fakhari and S. Yassemi, On the h-vector of a simplicial complex with Serre's condition, J. Pure Appl. Alg. 216 (2012), 91-94.

11. H. Haghighi, N. Terai, S. Yassemi and R. Zaare-Nahandi, Sequentially $S_{r}$ simplicial complexes and sequentially $S_{2}$ graphs, Proc. Amer. Math. Soc. 139 (2011), 1993-2005.

12. H. Haghighi, S. Yassemi and R. Zaare-Nahandi, Bipartite $S_{2}$ graphs are Cohen-Macaulay, Bull. Math. Soc. Sci. Math. Roum. 53 (2010), 125-132.

13. R. Hartshorne, Complete intersections in characteristic $p>0$, Amer. J. Math. 101 (1979), 380-383. 
14. J. Herzog and T. Hibi, Componentwise linear ideals, Nagoya Math. J. 153 (1999), 141-153.

15. , Distributive lattices, bipartite graphs and Alexander duality, J. Alg. Combin. 22 (2005), 289-302.

16. _ Monomial ideals, Springer-Verlag, London, Ltd., London, 2011.

17. J. Herzog, T. Hibi and X. Zheng, Dirac's theorem on chordal graphs and Alexander duality, Europ. J. Combin. 25 (2004), 949-960.

18. , Monomial ideals whose powers have a linear resolution, Math. Scand. 95 (2004), 23-32.

19. J. Herzog, T. Hibi and X. Zheng, Cohen-Macaulay chordal graphs, J. Combin. Theor. 113 (2006), 911-916.

20. J. Herzog, Y. Takayama and N. Terai, On the radical of a monomial ideal, Arch. Math. 85 (2005), 397-408.

21. G. Kalai, Algebraic shifting, Adv. Stud. Pure Math. 33 (2001), 121-163.

22. D. König, Über Graphen und ihre Anwendung auf Determinantentheorie und Mengenlehre, Math. Ann. 77 (1916), 453-465.

23. , Theory of finite and infinite graphs, Birkhauser Boston, Inc., Boston, 1990.

24. G. Lyubeznik, On the arithmetical rank of monomial ideals, J. Alg. 112 (1988), 86-89.

25. E. Miller and B. Sturmfels, Combinatorial commutative algebra, SpringerVerlag, New York, 2005.

26. N.C. Minh and N.V. Trung, Cohen-Macaulayness of monomial ideals and symbolic powers of Stanley-Reisner ideals, Adv. Math. 226 (2011), 1285-1306.

27. J.R. Munkres, Elements of algebraic topology, Addison-Wesley Publishing Company, Menlo Park, CA, 1984.

28. S. Murai and N. Terai, h-Vectors of simplicial complexes with Serre's conditions, Math. Res. Lett. 16 (2009), 1015-1028.

29. M.R. Pournaki, S.A. Seyed Fakhari and S. Yassemi, On the h-triangles of sequentially $\left(S_{r}\right)$ simplicial complexes via algebraic shifting, Ark. Mat. 51 (2013), $185-196$.

30. G.A. Reisner, Cohen-Macaulay quotients of polynomial rings, Adv. Math. 21 (1976), 30-49.

31. G. Rinaldo, N. Terai and K.I. Yoshida, Cohen-Macaulayness for symbolic power ideals of edge ideals, J. Alg. 347 (2011), 1-22.

32. , On the second powers of Stanley-Reisner ideals, J. Comm. Alg. 3 (2011), 405-430.

33. P. Schenzel, Dualisierende Komplexe in der lokalen Algebra und Buchsbaum-Ringe, Lect. Notes Math. 907, Springer-Verlag, New York, 1982.

34. A. Simis, On the Jacobian module associated to a graph, Proc. Amer. Math. Soc. 126 (1998), 989-997. 
35. A. Simis, W.V. Vasconcelos and R.H. Villarreal, On the ideal theory of graphs, J. Alg. 167 (1994), 389-416.

36. R.P. Stanley, Combinatorics and commutative algebra, Second Edition, Progr. Math. 41, Birkhauser Boston, Inc., Boston, 1996.

37. A. Taylor, The inverse Gröbner basis problem in codimension two, J. Symbol. Comp. 33 (2002), 221-238.

38. N. Terai, Alexander duality in Stanley-Reisner rings, Affine algebraic geometry, Osaka University Press, Osaka, 2007.

39. N. Terai and N.V. Trung, Cohen-Macaulayness of large powers of StanleyReisner ideals, Adv. Math. 229 (2012), 711-730.

40. N. Terai and K.I. Yoshida, A note on Cohen-Macaulayness of StanleyReisner rings with Serre's condition $\left(S_{2}\right)$, Comm. Alg. 36 (2008), 464-477.

41. A. Van Tuyl, Sequentially Cohen-Macaulay bipartite graphs: Vertex decomposability and regularity, Arch. Math. 93 (2009), 451-459.

42. R.H. Villarreal, Cohen-Macaulay graphs, Manuscr. Math. 66 (1990), 277293.

43. R. Woodroofe, Vertex decomposable graphs and obstructions to shellability, Proc. Amer. Math. Soc. 137 (2009), 3235-3246.

44. K. Yanagawa, Alexander duality for Stanley-Reisner rings and squarefree $\mathbb{N}^{n}$-graded modules, J. Alg. 225 (2000), 630-645.

Department of Mathematical Sciences, Sharif University of Technology, P.O. Box 11155-9415, Tehran, Iran, and School of Mathematics, Institute for Research in Fundamental Sciences (IPM), P.O. Box 19395-5746, Tehran, IRAN

\section{Email address: pournaki@ipm.ir}

School of Mathematics, Institute for Research in Fundamental Sciences (IPM), P.O. Box 19395-5746, Tehran, Iran

\section{Email address: fakhari@ipm.ir}

Department of Mathematics, Faculty of Culture and Education, Saga UNIVERSITY, SAGA 840-8502, JAPAN

Email address: terai@cc.saga-u.ac.jp

School of Mathematics, Statistics and Computer Science, College of Science, University of Tehran, Tehran, Iran, and School of Mathematics, Institute for Research in Fundamental Sciences (IPM), P.O. Box 193955746, Tehran, Iran

Email address: yassemi@ut.ac.ir 